\title{
CONTINUITY CONDITIONS FOR THE HILBERT TRANSFORM ON QUASI-HILBERT SPACES
}

\author{
A. ŠAHOVIĆ, F. VAJZOVIĆ AND S. PECO
}

\begin{abstract}
We give necessary and sufficient conditions for the continuity of the Hilbert transform on complex quasi-Hilbert spaces, i.e. on complex, reflexive, strictly convex Banach spaces with Gâteauxdifferentiable norm and with generalized inner product.
\end{abstract}

\section{INTRODUCTION}

In this paper $X$ is a complex quasi-Hilbert space, i.e. $X$ is a complex, reflexive, strictly convex Banach space with Gâteaux-differentiable norm and with quasi-inner product $(\cdot, \cdot)$ introduced in $[3]$ as

$$
(x, y):=\langle x, y\rangle-i\langle x, i y\rangle \text {. }
$$

Here

$$
\langle x, y\rangle:=\lim _{t \searrow 0} \frac{\|x+t y\|^{2}-\|x\|^{2}}{2 t} \text { for } x, y \in X
$$

$\|\cdot\|$ is a norm on $X$ (see [5]).

In papers [3] and [6] we observed the Hilbert transform on complex quasiHilbert space $X$ and we gave a connection between the Hilbert transform $H$ and the operator $A_{+}$(see Definition 1.3) on some set dense in $X$ (Theorem 1.5). In this paper we show that the operator $i A_{+}$being the infinitesimal generator of a bounded strongly continuous group of operators in $B(X)$ is both necessary and sufficient for the continuity of Hilbert transform on complex quasi-Hilbert space $X$ (Theorem 2.1).

First we recall some notations, basic notions and claims that we will need in this paper. Let $X$ be a complex Banach space, and let $B(X)$ denote the complex Banach algebra of all bounded linear operators on $X$.

2000 Mathematics Subject Classification. 46E30, 46C50, 47G10.

Key words and phrases. Banach space, Gâteaux-differentiable norm, generalized inner product, group of operators, Hilbert transform. 
Definition 1.1. If $U(t)$ is an operator function on the real axis $\mathbb{R}$ to the Banach algebra $B(X)$ satisfying the following conditions:

i) $U\left(t_{1}+t_{2}\right)=U\left(t_{1}\right) U\left(t_{2}\right), \quad\left(t_{1}, t_{2} \in \mathbb{R}\right)$,

ii) $U(0)=I, \quad(I$ - identity operator $)$,

then the family $U(t), t \in \mathbb{R}$ is called a one-parameter group of operators in $B(X)$. It is strongly continuous if it is continuous at the origin in the strong operator topology, i.e. if

$$
\left.\lim _{t \rightarrow 0} U(t) x=x \quad(x \in X) \text { (in the } X-\text { norm, shorter, in } X\right) .
$$

If, in addition, there exists a constant $M(M \geq 1)$ such that

$$
\|U(t)\| \leq M \text { for all } t \in \mathbb{R},
$$

then the strongly continuous group $U(t), t \in \mathbb{R}$ is said to be bounded.

The infinitesimal generator $A$ of the group $U(t), t \in \mathbb{R}$ is defined by

$$
A x:=\lim _{t \rightarrow 0} \frac{U(t) x-x}{x}
$$

for all $x \in X$ for which the last limit in norm exists. $A$ is a closed linear operator with dense domain $D(A)$ in $X$.

Definition 1.2. [1] Let $U(t), t \in \mathbb{R}$ be a strongly continuous group of operators in $B(X)$, and let $H_{\epsilon, N} \quad(0<\epsilon<N<\infty)$ be a continuous linear operator on $X$ defined by

$$
H_{\epsilon, N} x:=\frac{1}{\pi} \int_{\epsilon \leq|t| \leq N} \frac{U(t) x}{x} d t \quad(x \in X) .
$$

If

$$
\lim _{\substack{\epsilon \rightarrow 0 \\ N \rightarrow \infty}} H_{\epsilon, N} x
$$

exists in $X$, it is denoted by $H x$ and called the Hilbert transform of $x$, i. e.

$$
H x=\lim _{\substack{\epsilon \rightarrow 0 \\ N \rightarrow \infty}} H_{\epsilon, N} x .
$$

Definition 1.3. [3] The strong closure of the operator $A_{0}$ defined by

$$
A_{0} F_{a} x:=a F_{a} x-F_{a}^{2} x, x \in X, a \geq 0
$$

is called the positive square root of $-A^{2}$ and it is denoted by $A_{+}$.

The family of operators $F_{a}, a \geq 0$ was introduced in [6] as

$$
F_{a} x:=\lim _{\alpha \searrow 0} F_{a, \alpha} x, \quad x \in X, a \geq 0,
$$


where

$$
F_{a, \alpha} x:=\frac{1}{\pi i} \int_{0}^{a} d u \int_{\alpha+i 0}^{\alpha+i u}\left[\lambda R\left(\lambda^{2},-A^{2}\right)+\bar{\lambda} R\left(\bar{\lambda}^{2},-A^{2}\right] d \lambda,\right.
$$

where $\lambda=\alpha+i y, \quad i=\sqrt{-1}$. Here the resolvent of $-A^{2}$ is denoted by $R\left(\lambda^{2},-A^{2}\right)$, i.e.

$$
R\left(\lambda^{2},-A^{2}\right)=\left(\lambda^{2}-\left(-A^{2}\right)\right)^{-1} \in B(X),
$$

$-A^{2}$ is the infinitesimal generator of the bounded strongly continuous cosine operator function $C(t)$ defined by

$$
C(t):=\frac{U(t)+U(-t)}{2}, t \in \mathbb{R}
$$

where $U(t), t \in \mathbb{R}$ is a bounded strongly continuous group of operators in $B(X)$ with the infinitesimal generator $i A$.

Note that a family of bounded linear operators $F_{a}, a \geq 0$ exists for every bounded strongly continuous cosine operator function on $X$.

The following holds (proved in [2] and [3]):

1) The limit in the definition of operators $F_{a}, a \geq 0$ exists for all $x \in X$ and $a \geq 0$,

2) $F_{a} x=\frac{2}{\pi} \int_{0}^{\infty}\left(\frac{\sin a t}{t}\right)^{2} C(2 t) x d t=\frac{2 a}{\pi} \int_{0}^{\infty}\left(\frac{\sin a t}{t}\right)^{2} C\left(\frac{2 t}{a}\right) x d t$,

3) $\left\|F_{a}\right\| \leq a$ for all $a \geq 0$,

4) function $a \rightarrow F_{a}$ is strongly continuous on $[0,+\infty)$,

5) $\lim _{a \rightarrow+\infty} \frac{F_{a} x}{a}=x, \quad x \in X$,

6) $F_{a} F_{b} x=F_{b} F_{a} x=2 \int_{0}^{a} F_{u} x d u+(b-a) F_{a} x, x \in X, 0 \leq a \leq b$,

7)

$$
F_{a} x \in D\left(A^{2 k}\right), k=1,2,3, \ldots, a \geq 0, x \in X,
$$

8) $A^{2} F_{a} x=F_{a} A^{2} x$ for all $x \in D\left(A^{2}\right), a \geq 0$,

9)

$$
\text { the set } \bigcup_{a \geq 0} \overline{F_{a}(X)} \text { is dense in } X \text {. }
$$

10) $\left\langle x, A_{+} x\right\rangle \geq 0$ for all $x \in D\left(A_{+}\right)$,

11)

$$
A_{+}{ }^{2} x=A^{2} x \text { for all } x \in D\left(A^{2}\right) .
$$

More about operators $F_{a}, a \geq 0$ and $A_{+}$and their properties can be seen in [2], [3], [4] and [7]. We will need the following theorems (proved in [3] and $[7])$ :

Theorem 1.4. Let $X$ be a (complex) quasi-Hilbert space, and let $C(t), t \in \mathbb{R}$ be a bounded strongly continuous cosine operator function with the infinitesimal generator $A$. If the number 0 belongs to the point spectrum of the 
generator $A$, and if the set of all $x \in X$ for which $A x=0$ is denoted by $L$, then there exists the subspace $M$ of the space $X$ such that $X$ is a direct and orthogonal sum of subspaces $L$ and $M$ (i.e. every $x \in X$ can be written in a unique way in the form

$$
x=l \stackrel{*}{+} y, \quad l \in L, m \in M,
$$

and $(l, m)=0)$. The subspaces $L$ and $M$ have the following properties:

a) $C(t) x=x$ for all $x \in L$ and $t \in \mathbb{R}$

b) $M$ is invariant relative to all operators $C(t), t \in \mathbb{R}$,

c) 0 does not belong to the point spectrum of the restriction of the operator $A$ on the subspace $M$.

Theorem 1.5. Let $X$ be a (complex) quasi-Hilbert space. If $U(t), t \in \mathbb{R}$ is a strongly continuous group of isometries in $B(X)$ with the infinitesimal generator $i A$, and if $A_{+}$is the positive square root from $-A^{2}$, then the Hilbert transform $H$ is defined on some set dense in $X$, and

$$
\begin{aligned}
H A x & =i A_{+} x \text { for all } x \in D\left(A^{2}\right), \\
H A_{+} x & =i A x \text { for all } x \in D\left(A^{2}\right) .
\end{aligned}
$$

Theorem 1.6. Let $X$ be a (complex) quasi-Hilbert space. If $A$ is the infinitesimal generator of a bounded strongly continuous cosine operator function, then 0 does not belong to the residual spectrum of the operator $A$.

Theorem 1.7. Let $X$ be a (complex) quasi-Hilbert space, and let $U(t), t \in \mathbb{R}$ be a strongly continuous group of isometries in $B(X)$ with the infinitesimal generator $i A$. If 0 does not belong to the point spectrum of the operator $A^{2}$, then

$$
H^{2} x=-x \text { for all } x \in D\left(A^{2}\right),
$$

where $H$ is the Hilbert transform.

Observe that the set $D\left(A^{2}\right)$ is dense in $X$.

Remark 1.8. Let $X$ be a complex Banach space with quasi-inner product $(\cdot, \cdot)$ defined by $(1.1)$, and let $X^{*}$ be the dual space of space $X$. The following theorem holds (see [3] and [5]):

Given $\delta \in X^{*}$ there exists a unique $x_{\delta} \in X$ such that

$$
\left\|x_{\delta}\right\|=\|\delta\| \text { and }\left(x_{\delta}, y\right)=\delta(y) \text { for all } x \in X,
$$

and the mapping $\varphi: \delta \rightarrow x_{\delta}$ is continuous from the norm topology on $X^{*}$ to the weak topology on $X$ if and only if $X$ is a quasi-Hilbert space.

Set

$$
{ }^{*} \stackrel{*}{+} y:=\varphi\left(\varphi^{-1}(x)+\varphi^{-1}(y)\right), \quad x, y \in X .
$$

We have shown (see [3]): 
a) the space $X$ under the operation $\stackrel{*}{+}$ is isometrically isomorphic to $X^{*}$, which we denote by

$$
(X, \stackrel{*}{+})=\left(X^{*},+\right)
$$

b) if $X$ is a quasi-Hilbert space, then $X^{*}$ is also a quasi-Hilbert space.

c) if $U$ is an isometry in $B(X)$, then

$$
(x, U y)=\left(U^{-1} x, y\right), x, y \in X,
$$

and $U$ is a linear operator in space $(X,+)$ and in space $(X, \stackrel{*}{+})$.

\section{Continuity CONDitions for the Hilbert transform on QUASI-HILBERT SPACES}

Theorem 2.1. Let $X$ be a complex quasi-Hilbert space with the quasi-inner product $(\cdot, \cdot)$ defined by (1.1). Let $U(t), t \in \mathbb{R}$ be a group of isometries in $B(X)$ with the infinitesimal generator $i A$, and let $A_{+}$be the positive square root from $-A^{2}$. The Hilbert transform $H$ is a continuous linear operator on $X$ into itself if and only if the operator $i A_{+}$is the infinitesimal generator of a bounded strongly continuous group of operators in $B(X)$.

Proof. Suppose $i A_{+}$is the infinitesimal generator of the bounded strongly continuous group $U_{+}(t), t \in \mathbb{R}$ in $B(X)$. Set

$$
\|x\|_{1}:=\sup _{s \in \mathbb{R}}\left\|U_{+}(s) x\right\|, \quad x \in X .
$$

Note that $\|\cdot\|_{1}$ is a new norm on $X$, and that the norm $\|\cdot\|_{1}$ is equivalent to the norm $\|\cdot\|$. Using Remark 1.8, it is easy to prove that the space $X$ under the norm $\|\cdot\|_{1}$ denoted by $\left(X,\|\cdot\|_{1}\right)$ is a quasi-Hilbert space. Also it is easy to see that groups $U(t), t \in \mathbb{R}$ and $U_{+}(t), t \in \mathbb{R}$ are continuous groups of isometries in $B(X)$, where $X=\left(X,\|\cdot\|_{1}\right)$.

From here till the end of this part of the proof, let $X=\left(X,\|\cdot\|_{1}\right)$, and suppose that 0 does not belong to the point spectrum $\sigma_{p}\left(A^{2}\right)$ of $A^{2}$. Set

$$
\begin{aligned}
L^{\prime} & :=\left\{x \in X \mid U(t) x=U_{+}(t) x, \quad t \in \mathbb{R}\right\}, \\
L^{\prime \prime} & :=\left\{x \in X \mid U(t) x=U_{+}(-t) x, \quad t \in \mathbb{R}\right\} .
\end{aligned}
$$

It is easy to see that both $L^{\prime}$ and $L^{\prime \prime}$ are (closed) subspaces of $X$.

Furthermore, if $x_{0} \in L^{\prime} \cap L^{\prime \prime}$, then

$$
U(t) x_{0}=U(-t) x_{0},
$$

so

$$
\frac{U(t) x_{0}-x_{0}}{t}=\frac{U(-t) x_{0}-x_{0}}{t}, \quad t \neq 0 .
$$


Thus,

Since

$$
i A\left(\frac{1}{t} \int_{0}^{t} U(s) x_{0} d s-\frac{1}{t} \int_{0}^{t} U(-s) x_{0} d s\right)=0, \quad t \neq 0
$$

$$
\frac{1}{t} \int_{0}^{t} U(s) x_{0} d s-\frac{1}{t} \int_{0}^{t} U(-s) x_{0} d s \rightarrow 2 x_{0} \text { as } t \rightarrow 0
$$

and since $i A$ is a closed operator,

$$
x_{0} \in D(i A) \text { and } i A x_{0}=0 \text {, }
$$

so,

Since $0 \notin \sigma_{p}\left(A^{2}\right)$,

$$
-A^{2} x_{0}=0
$$

$$
L^{\prime} \cap L^{\prime \prime}=\{0\} .
$$

Using the properties of isometries in $B(X)$ and the definition of subspaces $L^{\prime}$ i $L^{\prime \prime}$, we get

$$
(x, U(t) y)=(U(-t) x, y)=\left(U_{+}(t) x, y\right)=\left(x, U_{+}(-t) y\right)=(x, U(-t) y)
$$

for all $t \in \mathbb{R}, x \in L^{\prime \prime}, y \in L^{\prime}$. Hence,

$$
(x, U(t) y)=(x, U(-t) y), t \in \mathbb{R}, x \in L^{\prime \prime}, y \in L^{\prime} .
$$

Since $L^{\prime}$ and $L^{\prime \prime}$ are invariant relative to all operators $U(t)$, and $U(-t), t \in$ $\mathbb{R}$, from the last equation, by replacing $y$ with $U(t) y$, we obtain

$$
(x, U(2 t) y)=(x, y), t \in \mathbb{R}, x \in L^{\prime \prime}, y \in L^{\prime} .
$$

Hence,

$$
\left(x, \frac{U(2 t) y-y}{2 t}\right)=0, t \in \mathbb{R}, x \in L^{\prime \prime}, y \in L^{\prime}
$$

Thus,

$$
(x, i A y)=0
$$

for all $x \in L^{\prime \prime}, y \in L^{\prime}, y \in D(A)$.

By Theorem 1.6, and since $0 \notin \sigma_{p}\left(A^{2}\right)$, the set $\left\{i A y \mid y \in L^{\prime}, y \in D(A)\right\}$ is dense in $L^{\prime}$. Hence,

$$
(x, y)=0 \text { for all } x \in L^{\prime \prime}, y \in L^{\prime} .
$$

Now, in the usual way, we get that every $l \in L, L:=\overline{L^{\prime} \dot{+} L^{\prime \prime}}$, can be written in a unique way in the form

$$
l=x+y, \quad x \in L^{\prime \prime}, y \in L^{\prime} .
$$

Let us prove that $L=X$.

First we shall prove that at least one of the spaces $L^{\prime}$ and $L^{\prime \prime}$ is not equal to $\{0\}$. 
For any $x \in K, \quad K:=\cup_{a \geq 0, b \geq 0} F_{a} F_{b}(X), \quad x \neq 0$ there exist $A_{+} A x$ and $A A_{+} x$, and

$$
A_{+} A x=A A_{+} x .
$$

By (1.2) and by (1.4),

$$
\left(A+A_{+}\right)\left(A-A_{+}\right) x=A^{2} x-A_{+}^{2} x=0, \quad x \in K .
$$

If $A x-A_{+} x=0$, then

$$
V_{1}(t) x:=U(t) U_{+}(-t) x
$$

is strongly differentiable with respect to $t$ on $\mathbb{R}$ and

$\frac{d V_{1}(t) x}{d t}=\frac{d U(t)}{d t} U_{+}(-t) x+U(t) \frac{d U_{+}(-t) x}{d t}=i U(t) U_{+}(-t)\left(A x-A_{+} x\right)=0$.

Thus,

$$
V_{1}(t) x=V_{1}(0) x \text { for all } t \in \mathbb{R},
$$

i.e. $x \in L^{\prime}$. So,

If $A x-A_{+} x \neq 0$, then

$$
L^{\prime} \neq\{0\}
$$

$$
y:=A x-A_{+} x \neq 0 \text { and } A y+A_{+} y=0 .
$$

The function

$$
V_{2}(t) y:=U(t) U_{+}(t) y, \quad t \in \mathbb{R}
$$

is strongly differentiable with respect to $t$ on $\mathbb{R}$ and

$$
\frac{d V_{2}(t) y}{d t}=0 .
$$

Thus, in a similar way, $y \in L^{\prime \prime}$. So,

$$
L^{\prime \prime} \neq\{0\} .
$$

Now, let us prove that $L=X$.

If $L \subset X, L \neq X$, and if the set of all $x \in X$ for which $(x, y)=$ 0 (for all $y \in L$ ) is denoted by $X_{1}$, then $X_{1} \neq\{0\}$, and $X_{1} \cap L=\{0\}$. Moreover

$$
X_{1} \cap\left(L^{\prime}+L^{\prime \prime}\right)=\{0\} .
$$

By Remark $1.8,\left(X_{1}, \stackrel{*}{+}\right)$ is a quasi-Hilbert space. $X_{1}$ is invariant relative to operators $U(t)$ and $U_{+}(t), t \in \mathbb{R} . U(t), t \in \mathbb{R}$ and $U_{+}(t), t \in \mathbb{R}$ are strongly continuous groups of isometries in $B\left(X_{1}\right)$. Hence, the claim we have already proved for space $X$ holds for $X_{1}$. That means that there exists $x_{1} \in X_{1}, \quad x_{1} \neq 0$ such that $x_{1} \in L^{\prime}$ or $x_{1} \in L^{\prime \prime}$. This is in contradiction 
with (2.1), proving that $L=X$.

From the definition of subspaces $L^{\prime}$ and $L^{\prime \prime}$ of $X$ we easily obtain

$$
\begin{aligned}
& A y=A_{+} y \text { for } y \in D(A) \cap L^{\prime}, \text { and } \\
& A x=-A_{+} x \text { for } x \in D(A) \cap L^{\prime \prime} .
\end{aligned}
$$

From this and from the Theorem 1.5, it follows that $H$ is a continuous linear operator on some set dense in $L=X$. Consequently, $H$ has a unique continuous linear extension on $X$ into itself, which completes this part of the proof.

Observe that in the above proof, we have used the assumption that $0 \notin$ $\sigma_{p}\left(A^{2}\right)$. In case $0 \in \sigma_{p}\left(A^{2}\right)$, the claim easily follows from the Theorem 1.4 together with the above proof.

Now, suppose that the Hilbert transform $H$ is a continuous linear operator on $X$ into itself. Then, by Theorem $1.7, H^{2}=-I$. From this it follows that the operator

$$
H^{\prime}:=i H
$$

is also a continuous linear operator and

$$
\left(H^{\prime}\right)^{2}=I .
$$

Lets prove that the group $U_{+}(t), t \in \mathbb{R}$ is bounded, where $U_{+}(t), t \in \mathbb{R}$ is a strongly continuous group of operators in $B(X)$ with the infinitesimal generator $i A_{+}$. Set

$$
P:=\frac{1}{2}\left(I+H^{\prime}\right), \quad Q:=\frac{1}{2}\left(I-H^{\prime}\right) .
$$

Operators $P$ and $Q$ are bounded, because the operator $H^{\prime}$ is bounded, and holds:

$$
P^{2}=P, \quad Q^{2}=Q, \quad P Q=Q P=0, \quad P+Q=I .
$$

Thus, every $x \in X$ can be written in a unique way in the form

$$
x=x^{\prime}+x^{\prime \prime}, x^{\prime}=P x, x^{\prime \prime}=Q x .
$$

From this immediately follows

$$
\left\|x^{\prime}\right\| \leq\|P\| \cdot\|x\| \text { and }\left\|x^{\prime \prime}\right\| \leq\|Q\| \cdot\|x\| .
$$

Let $x=F_{a} z, z \in X, a \geq 0$ be arbitrary, but fixed. Then

$$
x=x^{\prime}+x^{\prime \prime}, x^{\prime}=P x, x^{\prime \prime}=Q x,
$$

and

$$
\begin{gathered}
x^{\prime}=\frac{1}{2}\left(x^{\prime}+H^{\prime} x^{\prime}\right), x^{\prime} \in D(A), x^{\prime} \in D\left(A_{+}\right), \\
A H^{\prime} x^{\prime}=H^{\prime} A x^{\prime}, A_{+} H x^{\prime}=H A_{+} x^{\prime} .
\end{gathered}
$$


By Theorem 1.5. we have,

$$
i A x^{\prime}=\frac{1}{2}\left(i A x^{\prime}+i H^{\prime} A x^{\prime}\right)=\frac{1}{2}\left(i A x^{\prime}-H A x^{\prime}\right)=\frac{1}{2}\left(i A x^{\prime}-i A_{+} x^{\prime}\right),
$$

and

$$
i A_{+} x^{\prime}=\frac{1}{2}\left(i A_{+} x^{\prime}-H A_{+} x^{\prime}\right)=\frac{1}{2}\left(i A_{+} x^{\prime}-i A x^{\prime}\right) .
$$

Hence,

$$
i A x^{\prime}=-i A_{+} x^{\prime} .
$$

In a similar way it can be proved that

$$
i A x^{\prime \prime}=i A_{+} x^{\prime \prime} .
$$

The function

$$
W(s) x^{\prime \prime}:=U(t-s) U_{+}(s) x^{\prime \prime}
$$

is strongly differentiable with respect to $\mathrm{s}$ in $0<s<t$ for each fixed $t>0$ and

$$
\begin{aligned}
\frac{d W(s)}{d s} x^{\prime \prime} & =-\frac{d U(t-s)}{d(t-s)} \cdot U_{+}(s) x^{\prime \prime}+U(t-s) \cdot \frac{d U_{+}(s)}{d s} x^{\prime \prime}= \\
& =-U(t-s) U_{+}(s) i A x^{\prime \prime}+U(t-s) \cdot U_{+}(s) i A_{+} x^{\prime \prime} .
\end{aligned}
$$

From this and from (2.4) we obtain

$$
\frac{d W(s)}{d s} x^{\prime \prime}=U(t-s) U_{+}(s) \cdot\left(i A_{+} x^{\prime \prime}-i A x^{\prime \prime}\right)=0 .
$$

Thus,

which shows that

$$
W(s) x^{\prime \prime}=W(t) x^{\prime \prime}=W(0) x^{\prime \prime}
$$

$$
U(t) x^{\prime \prime}=U_{+}(t) x^{\prime \prime} \quad \text { for all } t \geq 0 .
$$

In a similar way it can be proved that

$$
U(t) x^{\prime \prime}=U_{+}(t) x^{\prime \prime} \text { for all } t<0 .
$$

Hence,

Similarly, by (2.3) we get

$$
U(t) x^{\prime \prime}=U_{+}(t) x^{\prime \prime} \quad \text { for all } t \in \mathbb{R} .
$$

$$
U(t) x^{\prime}=U_{+}(-t) x^{\prime} \text { for all } t \in \mathbb{R} .
$$

Thus,

$$
\begin{aligned}
U_{+}(t) x & =U_{+}(t) x^{\prime}+U_{+}(t) x^{\prime \prime}= \\
& =U(-t) x^{\prime}+U(t) x^{\prime \prime}
\end{aligned}
$$

for all $x \in \bigcup_{a \geq 0} \overline{F_{a}(X)}, x=x^{\prime}+x^{\prime \prime}, x^{\prime}=P x, x^{\prime \prime}=Q x$ and for all $t \in \mathbb{R}$. 
From this and from (2.2) it follows

$$
\begin{aligned}
\left\|U_{+}(t) x\right\| & \leq\left\|U(-t) x^{\prime}\right\|+\left\|U(t) x^{\prime \prime}\right\|=\left\|x^{\prime}\right\|+\left\|x^{\prime \prime}\right\| \leq \\
& \leq\|P\| \cdot\|x\|+\|Q\| \cdot\|x\|=(\|P\|+\|Q\|) \cdot\|x\|
\end{aligned}
$$

for all $x \in \bigcup_{a \geq 0} \overline{F_{a}(X)}$ and for all $t \in \mathbb{R}$. Since the set $\bigcup_{a \geq 0} \overline{F_{a}(X)}$ is dense in $X$ (by $(1.3)$ ),

$$
\left\|U_{+}(t) x\right\| \leq(\|P\|+\|Q\|) \cdot\|x\|
$$

for all $x \in X$ and for all $t \in \mathbb{R}$. By Definition 1.1, the group $U_{+}(t), t \in \mathbb{R}$ is bounded. The theorem is proved.

\section{REFERENCES}

[1] S. Ishikawa, Hilbert transforms on one-parameter groups of operators, Tokyo, J. Math., 9 (1986), 383-393.

[2] M. Radić and F. Vajzović, On the bounded cosine operator function in Banach space, Mat. Vesnik, 39 (1987), 187-204.

[3] A. Šahović and F. Vajzović, Cosine operator functions and Hilbert transforms, NSJOM, 35 (2) (2005), 41-55.

[4] A. Šahović and F. Vajzović, A spectrality condition for infinitesimal generators of cosine operator functions, Mat. Vesnik, 60 (2008), 193-206.

[5] R.A. Tapia, A characterization of inner product spaces, Proc. Amer. Math. Soc., 41 (1973), 569-574.

[6] F. Vajzović, On the cosine operator function, Glasnik Mat., 22 (42) (1987), 381-406.

[7] F. Vajzović and A. Šahović, Cosine operator functions and Hilbert transforms II, Proc. III Congress of Mathemataticians of Macedonia, Struga, Macedonia (2005), 347-356.

(Received: April 19, 2013)

(Revised: July 19, 2013)

\author{
A. Šahović and S. Peco \\ University "Džemal Bijedić" \\ USRC "M. Hujdur-Hujka" \\ 88000 Mostar \\ Bosnia and Herzegovina \\ amina.sahovic@unmo.ba \\ sead.peco@unmo.ba \\ F. Vajzović \\ Academy of Sciences and Arts of Bosnia \\ and Herzegovina (ANUBiH) \\ Bistrik 7, 71000 Sarajevo \\ Bosnia and Herzegovina
}

